\title{
Clinical observation of glucocorticoids application in patients with severe coronavirus disease 2019 (COVID-19): a descriptive study
}

\section{Linli Yue}

Maternal and Child Health Hospital of Hubei Province

\section{Xuan Wang}

Central Hospital of Wuhan

\section{Lefei Han}

The Hong Kong Polytechnic University

\section{Lin Yang}

The Hong Kong Polytechnic University

\section{Xiaolan Li}

Maternal and Child HEalth Hospital of Hubei Province

\section{Zhenzhen Wan}

Maternal and Child Health Hospital of Hubei Province

$\mathrm{Na}$ Li ( $\sim$ lina@hbfy.com )

Hubei Maternal and Children Health Hospital

Hong Jiang

Central Hospital of Wuhan

\section{Research article}

Keywords: COVID-19, glucocorticoids, complication, adverse effect, therapy

Posted Date: May 8th, 2020

DOI: https://doi.org/10.21203/rs.3.rs-25755/v1

License: (1) (1) This work is licensed under a Creative Commons Attribution 4.0 International License. Read Full License 


\section{Abstract \\ Background}

The coronavirus disease 2019 (COVID-19) caused an ongoing pandemic and bring heavy burden globally. We retrospectively investigated the effect of glucocorticoids on clinical symptoms, course of disease, and negative change of nucleic acid detection in patients with severe COVID-19.

\section{Methods}

Individual demographics data, CT images, clinical outcomes, laboratory investigations, treatment of patients with severe COVID-19 were collected from electronic medical records of the Central Hospital of Wuhan, from January 28 to February 28, 2020.

\section{Results}

Eighteen severe cases of COVID-19 received low-dose of glucocorticoid treatments. They were initially treated with $40 \mathrm{mg}$ methylprednisolone with gradually reduced doses and changed into oral prednisolone. The courses of glucocorticoid treatments ranged from 4 to 30 days. The pulmonary infections were absorbed to different degrees in 16 cases, one case deteriorated in CT imaging and one had no significant change. All cases had fever regression. Fifteen cases could respire without oxygen supply. Two were still inhaled oxygen through nasal catheters and one with high flow oxygen gradually transferred to face mask for oxygen supply. Five cases discharged. Twelve cases who were in stable conditions for treatment remained in hospital. One case was still in critical condition. The results of three re-examinations of SARS-Cov-2 nucleic acids by RT-PCR were negative.

\section{Conclusions}

The early use of low-dose glucocorticoid therapy in severe COVID-19 infection may have a positive effect on the prevention and treatment of disease deterioration.

\section{Introduction}

Since December 2019, the outbreak of the 2019 novel coronavirus disease (COVID-19) infection has spread rapidly and caused serious threats to China and the global world. As of April 24, there are more than 2.8 million cases confirmed for COVID-19 globally, and near 200,000 patients dead [1]. The causative pathogen was identified as SARS-CoV-2 which was genetically related to two previous highly pathogenic coronaviruses SARS-CoV (79\% similarity) and MERS-CoV (50\%) [2]. Epidemiological studies have found that SARS-CoV-2 had a higher transmission rate (basic reproductive number ranges from 2 to 
3) [3], and a varying case fatality rate across age groups (range from 0 in children to $14.8 \%$ in patients aged 80 years or over) [4].

Glucocorticoids has been widely used to treat severe acute respiratory infections by infectious pathogens. A retrospective cohort study showed that the proper use of glucocorticoids could reduce mortality and shorten the length of hospital stay for Severe Acute Respiratory Syndrome (SARS) [5]. However, the use of glucocorticoids remains controversial because of its adverse effects. Previous studies reported that glucocorticoids may be associated with the increased mortality and delaying viral clearance among SARS patients [6, 7]. The adverse effects were also observed in treatment of Middle East respiratory syndrome (MERS), including being more likely to receive invasive ventilation, increasing the risks of secondary infections and delaying clearances of MERS-CoV $[8,9]$. The guideline by World Health Organization (WHO) recommended not to routinely use systemic corticosteroids for patients with COVID19 pneumonia [10]. The guideline by National Health Commission of China also recommended the use of corticosteroids, but should fulfill strict criteria [11]. So far, the clinical report about the therapeutic and other effects on systemic glucocorticoid therapy among patients with COVID-19 remain sparse. A better understanding of the benefit and adverse effects of corticosteroids in treatment of CPVID-19 is critical. This study retrospectively evaluated the safety and efficacy of glucocorticoids therapy among patients with severe COVID-19 infections.

\section{Methods}

\section{Patients}

Medical records of patients with COVID-19 who were admitted into the Central Hospital of Wuhan were retrospectively retrieved from January 28 to February 28, 2020. We followed the clinical diagnosis criteria for COVID-19 by the guideline from the National Health Commission of China [12], which included 1) positive results of reverse transcription polymerase chain reaction (RT-PCR) tests for SARS-Cov-2 and 2) patchy shadows and ground glass opacity in chest computed tomography (CT) imaging. Patients who were classified as the severe type of COVID-19 pneumonia were included in the study. According to the classification criteria [12], server type patients should fulfill any of the following items: 1) Dyspnea, RR > 30 times/min; 2) Oxygen saturation $\left.\left(\mathrm{SaO}_{2}\right) \leq 93 \% ; 3\right)$ partial pressure of oxygen $\left(\mathrm{PaO}_{2}\right) /$ fraction of inspired oxygen $\left(\mathrm{FiO}_{2}\right) \leq 300 \mathrm{mmHg}$; or 4) Pulmonary imaging showed that the lesions progressed more than $50 \%$ within $24-48$ hours.

\section{Data Collection And Analysis}

We extracted the patients' records including demographic characteristics, clinical symptoms on admission, laboratory test on admission, chest CT imaging on admission and two to four times of reexamination, therapy information (duration, dosage and adverse effect) and outcomes. We summarized 
the continuous variable by mean and standard deviation or median and interquartile ranges (IQRs). Categorical variables were summarized by frequency and percentages.

\section{Results}

\section{Demographics and clinical manifestations}

Characteristics and clinical manifestations of patients were shown in Table 1. A total of 18 patients with 13 males were included. Their age ranged from 45 to 79 years. Among them, eight cases had hypertension, one case had both hypertension and diabetes and one case had both rheumatoid arthritis and bronchial asthma. Most patients had nonspecific clinical manifestations of COVID-19 pneumonia, including fever, dry cough, fatigue, chest pain, dyspnea, diarrhea, sore throat, etc. In our study, all patients felt asthenia. Sixteen patients (88.9\%) had fever. Thirteen patients $(72.2 \%)$ had cough. Diarrhea was observed in three cases (16.7\%). Six cases had dyspnea (33.3\%). Pharyngeal pain, nausea, and vomiting were observed in four patients. 
Table 1

Characteristics and clinical manifestations of patients $(\mathrm{N}=$ 18)

\begin{tabular}{|lll|}
\hline & N & $\%$ \\
\hline Gender (M) & 13 & $72.2 \%$ \\
\hline Age (45-79 years) $\geqq 65$ & 7 & $38.9 \%$ \\
\hline Co-morbidities & & \\
\hline Hypertension & 8 & $44.4 \%$ \\
\hline Diabetes + hypertension & 1 & $5.6 \%$ \\
\hline Rheumatoid arthritis + bronchial asthma & 1 & $5.6 \%$ \\
\hline Symptoms and signs & & \\
\hline Asthenia & 18 & $100.0 \%$ \\
\hline Fever & 16 & $88.9 \%$ \\
\hline Cough & 13 & $72.2 \%$ \\
\hline Shortness of breath & 13 & $72.2 \%$ \\
\hline Chest tightness & 12 & $66.7 \%$ \\
\hline Myalgia & 7 & $38.9 \%$ \\
\hline Dyspnoea & 6 & $33.3 \%$ \\
\hline Diarrhoea & 3 & $16.7 \%$ \\
\hline Vomiting & 2 & $11.1 \%$ \\
\hline Sore throat or pharyngalgia & 2 & $11.1 \%$ \\
\hline
\end{tabular}

\section{Laboratory findings}

Ten out of eighteen patients were positive in RT-PCR test for SARS-Cov-2 from their sputum specimen sample or nasopharynx swab. The other eight patients were positive on the second or the third PCR test. The laboratory results on admission were shown in Table 2. There were three cases of leucopenia in blood routine, and 17 cases (94.4\%) had decreases in the proportion of lymphocyte. D-dimer was increased in eleven patients (61.1\%). Transaminase increased in twelve patients and BNP (Brain Natriuretic Peptide) increased in five cases (45.4\%). 
Table 2

Laboratory test on admission of patients $(\mathrm{N}=18)$

\begin{tabular}{|lll|}
\hline & Mean & Standard deviation \\
\hline White blood cells $\left(\times 10^{9} / \mathrm{L}\right)$ & 5.3 & 2.4 \\
\hline Neutrophils $\left(\times 10^{9} / \mathrm{L}\right)$ & 4.1 & 2.2 \\
\hline Lymphocytes $\left(\times 10^{9} / \mathrm{L}\right)$ & 0.8 & 0.5 \\
\hline C-reactive protein $(\mathrm{mg} / \mathrm{dl})$ & 5.5 & 3.1 \\
\hline Interleukin-6 $(\mathrm{pg} / \mathrm{ml})$ & 5.9 & 9.4 \\
\hline Prothrombin time $(\mathrm{s})$ & 11.9 & 1.3 \\
\hline Activated partial thromboplastin time $(\mathrm{s})$ & 31.9 & 5.2 \\
\hline D-dimer $(\mu \mathrm{g} / \mathrm{ml})$ & 4.2 & 7.1 \\
\hline Creatine kinase $(\mathrm{U} / \mathrm{L})$ & 47.6 & 27.6 \\
\hline Creatine kinase muscle-brain & 5.5 & 2.1 \\
\hline isoform $(\mathrm{U} / \mathrm{L})$ & & \\
\hline Lactate dehydrogenase $(\mathrm{U} / \mathrm{L})$ & 237.1 & 65.0 \\
\hline Cardiac troponin $\mathrm{I}(\mu \mathrm{gg} / \mathrm{L})$ & 7.4 & 0.07 \\
\hline Brain natriuretic peptide $(\mathrm{pg} / \mathrm{ml})$ & 0.085 & 0.12 \\
\hline Alanine aminotransferase $(\mathrm{U} / \mathrm{L})$ & 102.7 & 116.9 \\
\hline Aspartate aminotransferase $(\mathrm{U} / \mathrm{L})$ & 36.8 & 29.7 \\
\hline Urea nitrogen $(\mathrm{mmol} / \mathrm{L})$ & 45.2 & 33.6 \\
\hline Creatinine $(\mu \mathrm{mol} / \mathrm{L})$ & 5.0 & 2.8 \\
\hline PH & 70.9 & 20.5 \\
\hline Partial Pressure of Carbon $\mathrm{Dioxide}(\mathrm{mmHg})$ & 41.7 & 6.2 \\
\hline Partial pressure of oxygen $(\mathrm{mmHg})$ & 75.8 & 10.9 \\
\hline Lactate $(\mathrm{mmol} / \mathrm{L})$ & 2.2 & 0.53 \\
\hline Blood glucose $(\mathrm{mmol} / \mathrm{L})$ & & \\
\hline
\end{tabular}

\section{CT imaging}

All the patients took chest CT scans on admission and took re-examination per week (on average two to four times of re-examination for each patients). On admission, all of 18 patients showed bilateral lung 
field with subpleural ground-glass spot shadow, with or without pleural thickening. After 1-2 weeks of the courses, nine of them deteriorated (50\%), often involving double lungs and multiple lobes, with asymmetric distribution under the pleura, and extending to the hilar, with pulmonary consolidation, bronchiectasis, even diffuse lesions in some critical patients. Most of the lung fields were involved by the lesions which were commonly known as "white lung". One patient did not have chest CT examination after 1-2 weeks of the course due to the critical condition. After active treatment, the infected lesions were absorbed in 16 patients (88.8\%) within 2-3 weeks. Five of them also had pulmonary consolidation (29.4\%) in parts of infected lesions at the same time and two had fibrosis (11.7\%) (Fig. 1). The results by imaging examination were not consistent with the clinical symptoms on the same patients. We observed some patients did not present obvious improvement of clinical symptoms, while their signs on the lung imaging still improved. Nevertheless, their overall outcomes were basically consistent.

\section{General treatment}

According to the diagnosis and treatment plan issued by the National Health Commission of China [11], the clinical status of patients were monitored, including duration of treatment, blood routine examination, biochemical indicators, coagulative function, lung imaging, oxygenation index, blood gas analysis, and inflammatory factors. The treatment plan followed antiviral (ribavirin, interferon, abidol), oxygen therapy, anti-secondary infection, anti-shock, water electrolyte acid-base balance and nutrition support, micro ecological balance treatment and traditional Chinese medicine treatment. Seventeen cases $(94.4 \%)$ were given oxygen therapy to improve hypoxemia during the treatments, including 16 patients had nasal catheter with oxygen $(2 \mathrm{~L} / \mathrm{min})$ and one patient had high flow oxygen $(30 \mathrm{~L} / \mathrm{min})($ Table 3$)$. 
Table 3

General treatment and adverse effect $(\mathrm{N}=18)$

No $\%$

Oxygen supply mode

High flow oxygen (flow 30L/min) $15.6 \%$

Nasal catheter (flow 2L/min) $\quad 16 \quad 88.9 \%$

No use $\quad 1 \quad 5.6 \%$

$\begin{array}{lll}\text { Antivirus } & 18 \quad 100.0 \%\end{array}$

Antibiotic $\quad 16 \quad 88.9 \%$

Chinese medicine $\quad 18 \quad 100.0 \%$

$\begin{array}{lll}\text { Anticoagulant therapy } & 13 \quad 72.2 \%\end{array}$

Drugs for liver protection $\quad 12 \quad 66.7 \%$

$\begin{array}{lll}\text { Gamma globulin } \quad 8 & 44.4 \%\end{array}$

Human albumin $\quad 16 \quad 88.9 \%$

Adverse effects

\begin{tabular}{|lll|}
\hline Gastrointestinal bleeding & 1 & $5.6 \%$ \\
\hline Insomnia & 5 & $26.3 \%$ \\
\hline Diarrhea & 2 & 11.1 \\
\hline
\end{tabular}

\section{Application of glucocorticoids}

According to the guideline [11], glucocorticoids could be used appropriately, if the lung imaging aggravates rapidly, oxygenation indicators are deteriorating, or patients with hyperactive inflammatory response. The indications for the use of glucocorticoids included: (1) Severe and critical diseases could be used at early stage; (2) continuous high fever ( $\left.>39^{\circ} \mathrm{C}\right)$; (3) CT imaging indicated infected lesions were large (more than $30 \%$ of the lobes are involved); (4) the CT imaging manifestations of the lungs were progressive deteriorated rapidly and the involved areas were significantly increasing; (5) Interleukin 6 (IL6) is significantly increased. The dose of glucocorticoids was suggested not to exceed $1-2 \mathrm{mg} / \mathrm{kg} / \mathrm{day}$ to avoid immunosuppressive, which may delay the clearance of viruses. In this study, all the 18 severe patients were treated with glucocorticoids, and their clinical indexes were monitored dynamically, including blood routine examination, biochemical, CT imaging changes, adverse reactions, and complications.

The dose of glucocorticoids matched the degree of inflammatory injury. In principle, the dosage of the methylprednisolone was halved in 3-5 days after the improvement of symptoms and signs, reduced to 
$20 \mathrm{mg} /$ day later and changed to oral prednisone, and gradually reduced to discontinuation in 3-5 days. The courses of the treatment were different according to the clinical conditions of the patients. In this study, four patients were given $40 \mathrm{mg}$ methylprednisolone, intravenous drip, twice per day (b.i.d) for on average 5.8 days. The other 14 patients were with the beginning dose of $40 \mathrm{mg}$ methylprednisolone per day (q.d) for 4 to 10 days, then reduced the dose to $30-20 \mathrm{mg} /$ day later and changed into oral prednisolone. The courses of the treatment were between 4 to 30 days and the median course was 17 days (Fig. 2). Proton pump inhibitors and calcium agents were used in all patients to prevent complications at the same time, and microecological regulators were used as appropriate. The examination of hepatitis $B$ and $C$ were tested in all patients to avoid potential infection before hormone therapy.

\section{Monitoring indicators}

Blood glucose, liver function, blood routine, C-reactive protein (CRP), imaging changes, electrolytes (especially blood potassium) were monitored during the treatment period. In case of any abnormality, patients should be treated as soon as possible, if the patients with sleep disorder, antipsychotic tablets should be given to improve their sleep. Six patients (33.3\%) present adverse effects, including insomnia in five patients, gastrointestinal bleeding in one patient (the patient also had insomnia), and diarrhea in two patients (one patient also had insomnia) (Table 3). Their symptoms were relieved after adjusting the dosage and expectant treatment. No other severe adverse effects were observed.

\section{Outcomes}

By 28 February 2020, the pulmonary infections were absorbed in 16 patients (88.9\%). One had no significant changes of CT imaging. One was deteriorated in CT imaging. All patients had fever regression. Fifteen patients (88.2\%) could respire without oxygen supply (Table 4). One patient with high flow oxygen gradually transferred to face mask for oxygen supply, and the oxygen saturation could be stable between 90-94\%. Two patients were still inhaled oxygen through nasal catheters. Eleven patients $(61.1 \%)$ had no respiratory symptoms after treatment. Six patients (33.3\%) had mild respiratory symptoms, such as cough, shortness of breath and chest tightness. One had moderate shortness of breath. No patients developed acute respiratory distress syndrome (ARDS). No fatal case was developed. All patients were negative for SARS-Cov-2 in three times of RT-PCR test. Five cases discharged according to the clinically standard of the guideline. Twelve cases were in stable conditions for treatment and remained in hospital. One case was still in critical condition. 
Table 4

Clinical manifestations after treatment $(\mathrm{N}=18)$

\begin{tabular}{|lll|}
\hline & Median & IQR \\
\hline Day of fever reduction & 4 & 2,5 \\
\hline Respiratory symptom & No & $\%$ \\
\hline No & & \\
\hline Mild (e.g. cough, shortness of breath, chest tightness) & 6 & $33.3 \%$ \\
\hline Moderate (shortness of breath) & 11 & $5.6 \%$ \\
\hline Breath without oxygen supply & 15 & $83.3 \%$ \\
\hline
\end{tabular}

\section{Discussion}

The pandemic of COVID-19 in 2020 has brought serious threats to the public around the world. As of April 24 2020, the crude global case fatality rate was around 7\% and largely variated from different countries [1]. So far, there are still no specific antiviral treatments for COVID-19 pneumonia. It becomes crucial issues for scientists to improve the cure rate, shorten the course of the severe type, and reduce the mortality caused by SARS-CoV-2 virus.

In our study, we retrospectively evaluated the benefit and harm on the use of glucocorticoids in patients with severe COVID-19. A total of 18 patients with severe COVID-19 pneumonia were treated with glucocorticoids. The courses of glucocorticoids therapy for patients were different according to their clinical conditions, which ranged from 4 to 30 days with a median course of 17 days. No severe adverse effects were observed. Six patients (33.3\%) presented mild adverse effects, including diarrhea, gastrointestinal bleeding and insomnia. Those symptoms were relived after adjusting the dosage and expectant treatment. Adverse effects associated with long-term glucocorticoids use are not considered relevant for this study, because of the short duration and low dose of the glucocorticoids prescribed.

The outcomes of our patients were generally satisfied. Sixteen patients had absorption of pulmonary infections. Fifteen patients could respire without oxygen supply. All the patients had three times of RTPCR test for SARS-CoV-2 after treatment and all patients were negative for the last time results. Five cases discharged according to clinically standard of the guideline. Twelve cases who were in a stable condition for treatment remained in hospital. One case was still in critical condition.

The use of corticosteroid remains controversial in treatment of respiratory infections and there is no conclusive evidence suggesting the balance of benefit and harms in treatment of COVID-19 pneumonia. Among severe patients with virus infection, the overwhelming inflammation and cytokine-related lung injury might cause rapidly progressive pneumonia, even fatal outcomes [13]. Corticosteroid has been 
widely used in clinic to decrease the inflammatory responses in lungs, prevent acute lung injury and avoid ARDS. However, the clinical guidelines of WHO [10] and the US [14] suggest against the routine corticosteroid therapy in patients with COVID-19 due to the concerns of adverse effects. A systematic review and meta-analysis from 15 studies showed that corticosteroid was associated with increased mortality, longer length of hospital stay and higher risk of secondary infections in critical patients with coronavirus infection [15]. However, the meta-analysis may be limited to enroll retrospective studies, remain controlling for the time and dosage of corticosteroid and a potential publication bias. In a prospective cohort study on hospitalization of influenza A (H1N1)pdm09, low-to-moderate dose corticosteroids (25-150 mg/d) was associated with reduced mortality among patients with $\mathrm{PaO}_{2} / \mathrm{FiO}_{2}<$ $300 \mathrm{mmHg}$, while the relationship was not observed in high dose group (>150 mg/d). The evidence also did not support the benefit of corticosteroid among mild patients $\left(\mathrm{PaO}_{2} / \mathrm{FiO}_{2}>300 \mathrm{mmHg}\right)$ [16]. Another RCT also supported the early, low-dose $(0.5 \mathrm{mg} / \mathrm{kg} / \mathrm{d})$ and short-term ( 5 or 7 days) use of corticosteroid therapy reduced the risk of treatment failure among patients with severe community-acquired pneumonia [17].

Our study added some evidence to precautiously consider the early use of low dose glucocorticoids to prevent fatal outcomes for severe patients with COVID-19. This is accordance with current limited evidence. A previous report with 15 severe COVID-19 patients suggested the early systematic corticosteroids therapy among could enhance $\mathrm{SaO}_{2}$ and $\mathrm{PaO}_{2} / \mathrm{FiO}_{2}$, and no evidence support the improvement on deaths due to the small sample size [18]. Another retrospective study with 11 of 31 patients received corticosteroid treatment reported that corticosteroid did no increase virus clearance time and length of hospital stay in patients with mild COVID-19 [19]. Nevertheless, corticosteroid therapy still requires carefully considering the patients' clinical conditions, time, dose, and duration of treatment. A well-designed large scale RCT is also warranted to further evaluate the efficacy of corticosteroid.

Our study has a few limitations. First, glucocorticoids treatments in our hospital were only applied for severe patients. Evidence from a small sample size with 18 patients might not be conclusive. Second, this was an observational study. We were not able to set up a referent group to compare the effect of glucocorticoids treatment. Confounding factors might not be eliminated. The delay of virus clearance, shorten of clinical courses and effect of reducing ARDS remained further investigation. Lastly, we only investigated the short-term effect of glucocorticoids treatments, a longer follow-up period is warranted for evaluate the long-term benefit in the future.

In summary, early appropriate use of glucocorticoids may benefit for patients with severe COVID-19 pneumonia. We did not observe adverse effects on the negative transformation of nucleic acid detection of COVID-19.

\section{Abbreviations}

ARDS: Acute respiratory distress syndrome; COVID-19, The coronavirus disease 2019; CRP, C-reactive protein; $\mathrm{CT}$, computed tomography; $\mathrm{FiO}_{2}$, fraction of inspired oxygen; IQRs, interquartile range; MERS, 
Middle East respiratory syndrome; $\mathrm{PaO}_{2}$, partial pressure of oxygen; RT-PCR, reverse transcription polymerase chain reaction tests; SARS, Severe Acute Respiratory Syndrome; $\mathrm{SaO}_{2}$, oxygen saturation; WHO, World Health Organization.

\section{Declarations}

\section{Ethics approval and consent to participate}

The ethical approval has been obtained from the Ethics Committee of the Central Hospital of Wuhan. The requirement for written informed consent of patients was waived by the Ethics Committee.

\section{Consent for publication}

Not applicable.

\section{Availability of data and materials}

The datasets used and/or analyzed during the current study are available from the corresponding author on reasonable request.

\section{Patient data}

The patients included in our study have not been reported in any other submission.

\section{Competing interests}

The authors report no potential conflicts of interest.

\section{Funding}

NL is supported by the Joint Fund of the Hubei Provincial Health Commission. LYang is supported by the Alibaba (China) Co., Ltd. Collaborative Fund and the General Research Fund of the Hong Kong Polytechnic University.

\section{Authors' Contributions}

LYue, NL and XW contributed to the conception and design of the study. XL and ZW contributed to data collection and clean. LH conducted data analysis. LYue, LH, NL and XW interpreted the findings and drafted the manuscript. LH, LYang, NL and HJ reviewed and edited the manuscript. All the authors proved the final version of this manuscript.

\section{Acknowledgements}

Not applicable 


\section{Authors' Information}

${ }^{1}$ Department of Anaesthesiology, Maternal and Child Health Hospital of Hubei Province, Hubei, China; ${ }^{2}$ Department of pediatrics, Central Hospital of Wuhan, Hubei, China; ${ }^{3}$ School of Nursing, The Hong Kong Polytechnic University, Hong Kong, China.

\section{References}

1. Coronavirus COVID-19 Global Cases by the Center for Systems Science and Engineering (CSSE) at Johns Hopkins University. Available at: https://www.arcgis.com/apps/opsdashboard/index.html\#/bda7594740fd40299423467b48e9ecf6. Accessed 8 April, 2020.

2. Lu R, Zhao X, Li J, Niu P, Yang B, Wu H, Wang W, Song H, Huang B, Zhu N, et al. Genomic characterisation and epidemiology of 2019 novel coronavirus: implications for virus origins and receptor binding. Lancet. 2020;395(10224):565-74. https://doi.org/10.1016/s0140-6736(20)302518.

3. Zhao S, Lin Q, Ran J, Musa SS, Yang G, Wang W, Lou Y, Gao D, Yang L, He D, et al. Preliminary estimation of the basic reproduction number of novel coronavirus (2019-nCoV) in China, from 2019 to 2020: A data-driven analysis in the early phase of the outbreak. Int J Infect Dis. 2020;92:214-7. https://doi.org/10.1016/j.ijid.2020.01.050.

4. Wu ZY, McGoogan JM. Characteristics of and Important Lessons From the Coronavirus Disease 2019 (COVID-19) Outbreak in China Summary of a Report of 72314 Cases From the Chinese Center for Disease Control and Prevention. JAMA. 2020;323(13):1239-42. https://doi.org/10.1001/jama.2020.2648.

5. Chen RC, Tang XP, Tan SY, Liang BL, Wan ZY, Fang JQ, Zhong N. Treatment of severe acute respiratory syndrome with glucosteroids: the Guangzhou experience. Chest. 2006;129(6):1441-52. https://doi.org/10.1378/chest.129.6.1441.

6. Auyeung TW, Lee JS, Lai WK, Choi CH, Lee HK, Lee JS, Li PC, Lok KH, Ng YY, Wong WM, et al. The use of corticosteroid as treatment in SARS was associated with adverse outcomes: a retrospective cohort study. J Infect. 2005;51(2):98-102. https://doi.org/10.1016/j.jinf.2004.09.008.

7. Lee N, Allen Chan KC, Hui DS, Ng EK, Wu A, Chiu RW, Wong VW, Chan PK, Wong KT, Wong E, et al. Effects of early corticosteroid treatment on plasma SARS-associated Coronavirus RNA concentrations in adult patients. J Clin Virol. 2004;31(4):304-9. https://doi.org/10.1016/j.jcv.2004.07.006.

8. Arabi YM, Mandourah Y, Al-Hameed F, Sindi AA, Almekhlafi GA, Hussein MA, Jose J, Pinto R, Al-Omari A, Kharaba A, et al. Corticosteroid Therapy for Critically III Patients with Middle East Respiratory Syndrome. Am J Respir Crit Care Med. 2018;197(6):757-67. https://doi.org/10.1164/rccm.201706$11720 \mathrm{C}$. 
9. Alfaraj SH, Al-Tawfiq JA, Assiri AY, Alzahrani NA, Alanazi AA, Memish ZA. Clinical predictors of mortality of Middle East Respiratory Syndrome Coronavirus (MERS-CoV) infection: A cohort study. Travel Med Infect Dis. 2019;29:48-50. https://doi.org/10.1016/j.tmaid.2019.03.004.

10. World Health Organization. Clinical management of severe acute respiratory infection (SARI) when COVID-19 disease is suspected: Interim guidance. Available at: https://www.who.int/publicationsdetail/clinical-management-of-severe-acute-respiratory-infection-when-novel-coronavirus-(ncov)infection-is-suspected.

11. National Health Commission of China. The notice of launching guideline on diagnosis and treatment of the novel coronavirus pneumonia (NCP). 5th edition. Available at: http://www.gov.cn/zhengce/zhengceku/2020-02/05/content_5474791.htm, 2020.

12. National Health Commission of China. New coronavirus pneumonia prevention and control program. Available at:

http://www.nhc.gov.cn/yzygj/s7653p/202002/8334a8326dd94d329df351d7da8aefc2.shtml. Accessed 22 February 2020.

13. Channappanavar R, Perlman S. Pathogenic human coronavirus infections: causes and consequences of cytokine storm and immunopathology. Semin Immunopathol. 2017;39(5):529-39. https://doi.org/10.1007/s00281-017-0629-x.

14. Infectious Diseases Society of America Guidelines on the Treatment and Management of Patients with COVID-19. Available at: https://www.idsociety.org/practice-guideline/covid-19-guidelinetreatment-and-management/. Accessed April 14, 2020.

15. Yang Z, Liu J, Zhou Y, Zhao X, Zhao Q, Liu J. The effect of corticosteroid treatment on patients with coronavirus infection: a systematic review and meta-analysis. J Infect. 2020. https://doi.org/10.1016/j.jinf.2020.03.062.

16. Li H, Yang SG, Gu L, Zhang Y, Yan XX, Liang ZA, Zhang W, Jia HY, Chen W, Liu M, et al. Effect of lowto-moderate-dose corticosteroids on mortality of hospitalized adolescents and adults with influenza A(H1N1)pdm09 viral pneumonia. Influenza Other Respir Viruses. 2017;11(4):345-54. https://doi.org/10.1111/irv.12456.

17. Torres A, Sibila O, Ferrer M, Polverino E, Menendez R, Mensa J, Gabarrus A, Sellares J, Restrepo MI, Anzueto $A$, et al. Effect of corticosteroids on treatment failure among hospitalized patients with severe community-acquired pneumonia and high inflammatory response: a randomized clinical trial. JAMA. 2015;313(7):677-86. https://doi.org/10.1001/jama.2015.88.

18. Zhou W, Liu Y, Tian D, Wang C, Wang S, Cheng J, Hu M, Fang M, Gao Y. Potential benefits of precise corticosteroids therapy for severe 2019-nCoV pneumonia. Signal transduction targeted therapy. 2020;5(1):18. https://doi.org/10.1038/s41392-020-0127-9.

19. Zha L, Li S, Pan L, Tefsen B, Li Y, French N, Chen L, Yang G, Villanueva EV. Corticosteroid treatment of patients with coronavirus disease 2019 (COVID-19). Med J Aust. 2020. https://doi.org/10.5694/mja2.50577. 
Figures

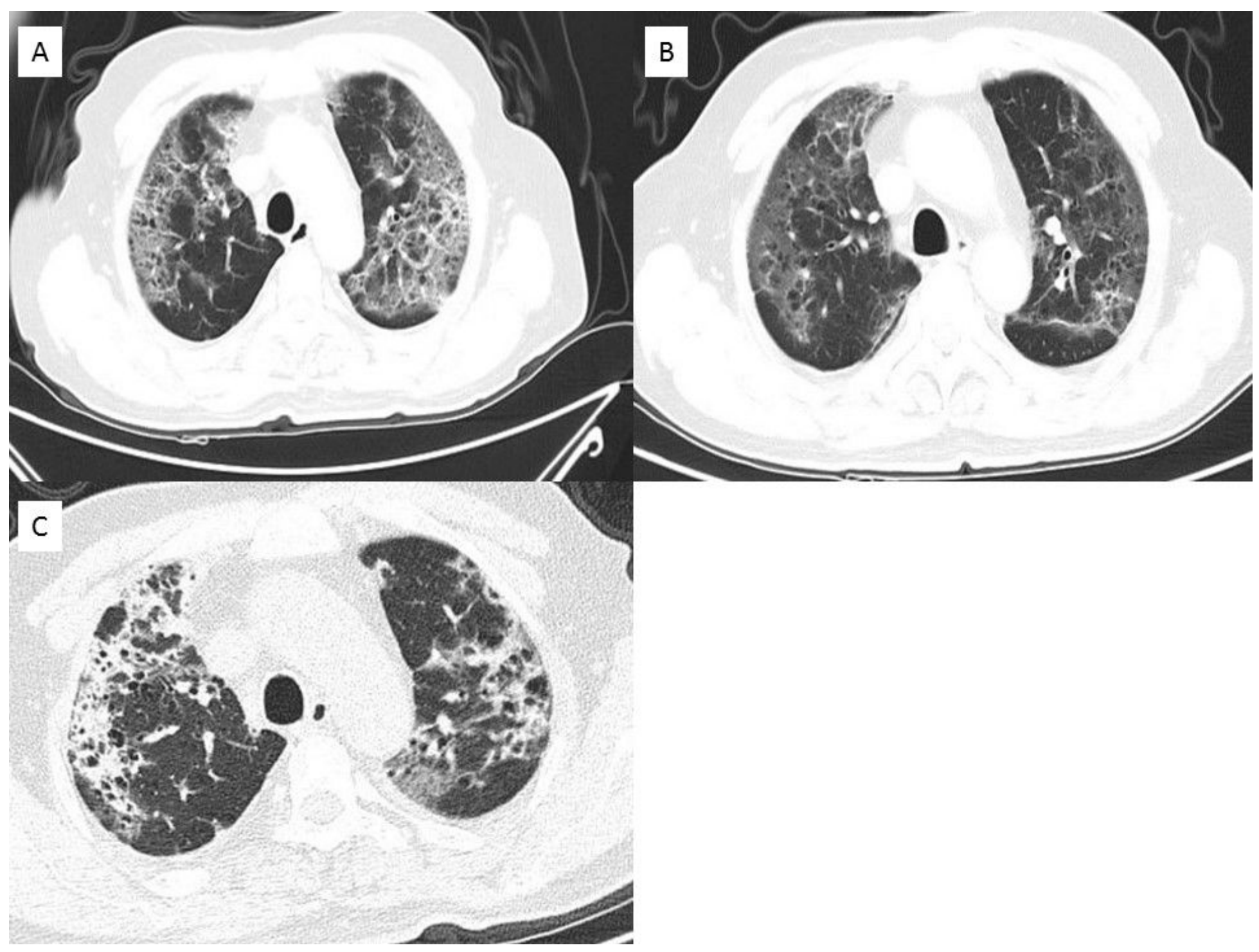

Figure 1

CT imaging obtained in a male aged ranged $65-70$ years with COVID-19 pneumonia. A: day 21 after symptom onset, multiple spot infections in double lungs. B: day 25 after symptom onset, double lung multiple spot infections were deteriorated, the scope was expanded, some lesions consolidated, a small amount of pleural effusion. C: day 35 after symptom onset, infective focus absorbed partially, and the original pleural effusion was absorbed. 


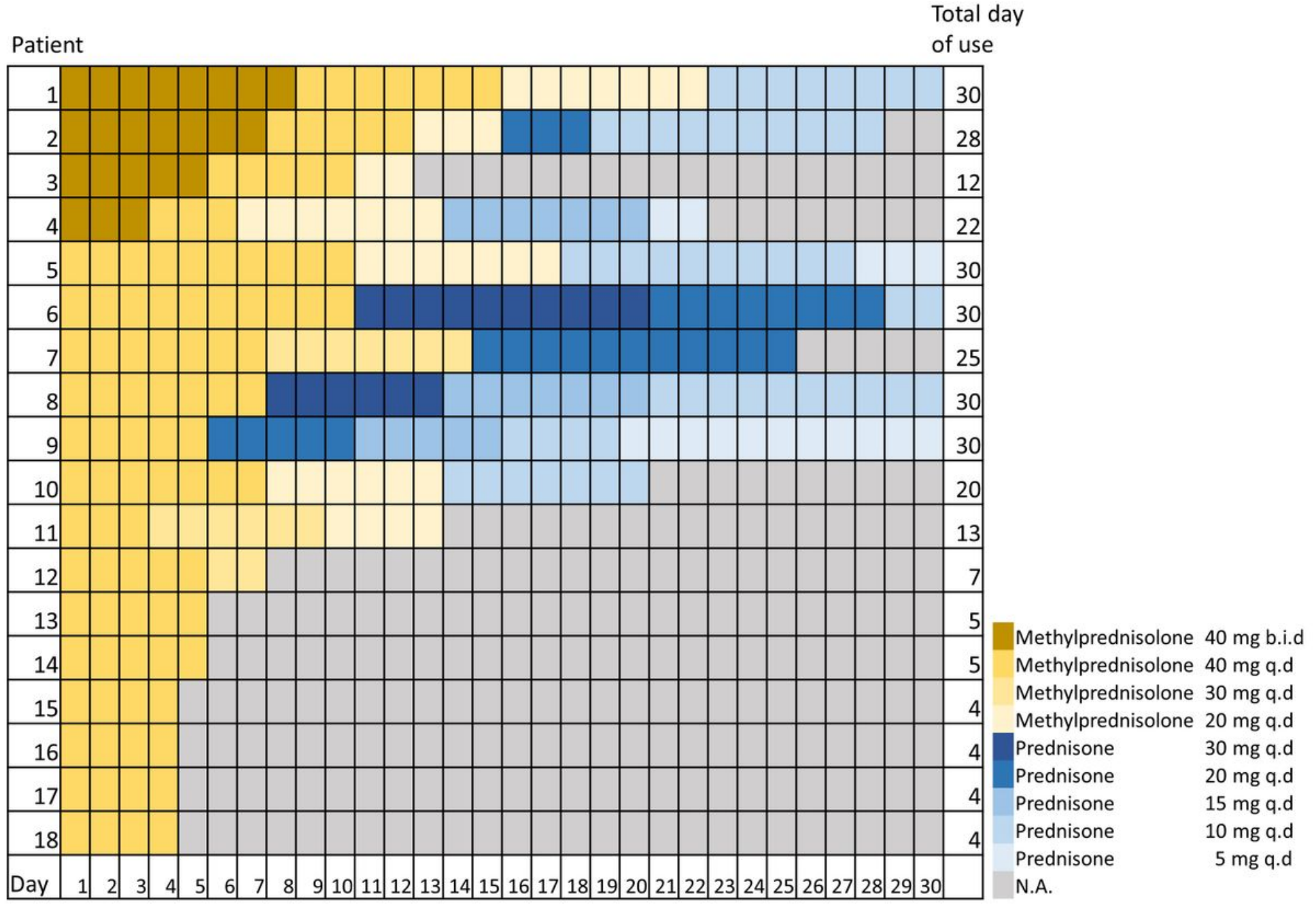

Figure 2

Application, duration and doses of glucocorticoids among 18 patients. Abbreviation: q.i.d, once per day; b.i.d, twice per day. 